\title{
BMJ Open Comorbidities and the use of comedications in people living with HIV on antiretroviral therapy in Japan: a cross-sectional study using a hospital claims database
}

To cite: Ruzicka DJ, Imai K, Takahashi K, et al. Comorbidities and the use of comedications in people living with HIV on antiretroviral therapy in Japan: a crosssectional study using a hospital claims database. BMJ Open 2018;8:e019985. doi:10.1136/ bmjopen-2017-019985

- Prepublication history and additional material for this paper are available online. To view these files, please visit the journal online (http://dx.doi. org/10.1136/bmjopen-2017019985).

Received 11 October 2017 Revised 26 April 2018 Accepted 14 May 2018

Check for updates

${ }^{1}$ Medical Affairs, MSD K.K. Tokyo, Japan

${ }^{2}$ Oncology Clinical Development, Merck \& Co., Inc., Kenilworth, New Jersey, USA

${ }^{3}$ Japan Development, MSD K.K., Tokyo, Japan

${ }^{4}$ Department of General

Medicine, Juntendo University, Tokyo, Japan

Correspondence to

Daniel J Ruzicka;

daniel.ruzicka@merck.com

\section{ABSTRACT}

Objectives To investigate the prevalence of chronic comorbidities and the use of comedications in people living with HIV (PLWH) and on antiretrovirals in Japan, by using a hospital claims database.

Design Observational, retrospective, cross-sectional study.

Setting A hospital claims database of Japanese hospitals that have advanced medical treatment capabilities (ie, advanced treatment hospitals, general hospitals, acute care hospitals), which include those providing acute and chronic care (excluding nursing homes or hospices).

Participants A total of 1445 PLWH aged $\geq 18$ years and with a prescription record of antiretrovirals between January 2010 and December 2015 were identified from the database.

Outcome measures The number and types of chronic comorbidities, as well as the number and types of comedications, in different age groups of the PLWH. Results The median (range) age of patients was 45 (18-90) years, and $90.4 \%$ were men. Of the 1445 patients, $972(67.3 \%)$ had at least one chronic comorbidity. Common chronic comorbidities included lipid disorders (31.6\%), diabetes (26.8\%), hypertension (18.2\%) and hepatitis B/C coinfection (18.2\%). Patients in the older age groups had greater numbers of chronic comorbidities. The most common chronic comorbidities in the older age groups were hypertension, diabetes and lipid disorders. The majority of patients used at least one comedication, and those in the older age groups used greater numbers of comedications. The most common therapeutic category of comedication included antacids, antiflatulents and antiulcerants (31.7\%). Of 151 malignancies reported in 117 patients, 84 were AIDS-defining cancers and 67 were non-AIDS-defining cancers.

Conclusions Chronic comorbidities and comedications were common among PLWH in Japan taking antiretrovirals; particularly among older patients, who more frequently used comedications. This suggests the need for giving special attention to the appropriate management of this patient population.
Strengths and limitations of this study

- This is the first study to examine chronic comorbidities and comedications in people living with HIV in Japan taking antiretrovirals, using a large-scale hospital claims database.

- Patients were stratified into small age groups, which provided a clear picture of patients' comorbidity and comedication profiles by age.

- The cross-sectional study design prevented inference of causality between HIV infection and chronic comorbidities or comedications.

- This study has limitations inherent in the use of a hospital claims database, such as poorly recorded or missing information, which may result in misclassification.

- The generalisability of the study results may be limited because data contained in the database were collected from Japanese hospitals that can provide advanced medical treatment to patients, not from all the hospitals providing HIV treatment in Japan.

\section{INTRODUCTION}

Improvements in antiretroviral therapy (ART) over the last 15-20 years have prolonged the life expectancy of people living with HIV (PLWH). Life expectancy of PLWH is now reportedly similar to that of people without HIV. ${ }^{1}$ However, in addition to HIV-related conditions, age-associated chronic comorbidities negatively affect the mortality rates of PLWH, as shown in various studies. ${ }^{2-6}$ Considering their extended lifespans, some chronic comorbidities among older PLWH may be partly attributed to normal age-related changes in physiological functioning, ${ }^{7}$ as with older people in general. Moreover, a longer duration of ART, which leads to increased toxicity, and HIV-induced persistent immunodeficiency and inflammation may also contribute to the increased number of comorbidities or higher prevalence of certain 
comorbidities among older PLWH. ${ }^{8-11}$ Chronic inflammation and immune system changes by HIV infection were also found potentially involved in the mechanisms for possible premature ageing in PLWH. ${ }^{12}$

Studies have shown that over half of all HIV deaths are caused either by coinfection or age-associated non-infectious chronic comorbidities, including vascular diseases, hypertension, lipid disorders, diabetes mellitus, kidney diseases, malignancies and bone disorders. ${ }^{13}{ }^{14}$ Such age-associated chronic comorbidities in PLWH have presented a new challenge for HIV treatment, especially in an ageing society such as Japan, which is among the countries with the longest life expectancy, for both sexes. ${ }^{15}$ In Japan, more than $10 \%$ of newly diagnosed people with HIV in 2015 were aged $\geq 50$ years, ${ }^{16}$ indicating a number of PLWH are relatively older. Although the majority of newly diagnosed people with HIV were aged $20-49$ years $(88.2 \%),{ }^{16}$ these people are also able to live to old age through appropriate management with antiretrovirals. Thus, in line with the trends in other countries, in which the proportions of PLWH aged $\geq 50$ years have increased, ${ }^{17}$ the number of older PLWH may also be increasing in Japan. Because of the potentially long lifespans of PLWH in Japan, it is important to obtain real-world data regarding the comorbidity profiles of these people.

Despite the importance of the association of chronic comorbidities with mortality rates among PLWH, only a few small studies on chronic comorbidities among PLWH in Japan have been published to date, ${ }^{18} 19$ and there have been no large multicentre epidemiological studies of chronic comorbidities in this population. Therefore, in this study to better understand a patient population, we examined the frequency and type of chronic comorbidities of interest and the use of comedications in different age groups of PLWH in Japan taking antiretrovirals and who were aged $\geq 18$ years, using a Japanese hospital claims database.

\section{METHODS \\ Study design}

This was an observational, retrospective, cross-sectional database study. Study data were extracted from a hospital claims database of acute care hospitals in Japan, compiled by Medical Data Vision (MDV, Tokyo, Japan). This electronic record-based hospital claims database contained information from approximately 17 million patients in 288 acute care hospitals (as of January 2017) in Japan (https://www.mdv.co.jp/press/2017/detail_743.html); accounting for approximately $17 \%$ of all acute care hospitals in Japan that use the diagnosis procedure combination/per-diem payment system. It should be noted, however, that 'acute care hospitals' included in this database are not merely acute care-only or emergency hospitals. It describes hospitals with advanced medical treatment capabilities (ie, advanced treatment hospitals, general hospitals, acute care hospitals), so that hospitals providing both acute and chronic care (excluding nursing homes or hospices) are also included in the database. Because this terminology is highly specific to Japan, it may be confusing for readers outside Japan or not familiar with the MDV database. Therefore, we refer to these hospitals included simply as 'hospitals' hereinafter. We used this database because in Japan PLWH usually receive treatment at regional hospitals for HIV/AIDS treatment, and such designated hospitals are generally large and provide advanced medical treatment for patients with acute and chronic diseases. The majority of patients in the database are aged $15-64$ years $(51.5 \%)$, followed by $\geq 65$ years $(34.2 \%)$ and $0-14$ years $(14.3 \%)$. The database contains both inpatient and outpatient data from any department; these data include age, sex, department visited, date of medical service, diagnosis codes, hospitalisation (if any), medical procedures and prescriptions.

\section{Study population}

PLWH, aged $\geq 18$ years, with a diagnosis record of HIV, and with a prescription record of antiretrovirals between January 2010 and December 2015 (study period) were included in the study. A person with any diagnosis record of HIV was identified by the presence of at least one record of the International Classification of Diseases, 10th Revision (ICD-10) codes B20-24: HIV disease resulting in infectious and parasitic diseases (B20), malignant neoplasms (B21), other specified diseases (B22), other conditions (B23) and unspecified HIV disease (B24). The patients were required to have at least one prescription record of any of the following antiretrovirals anytime during the study period: nucleoside reverse transcriptase inhibitors (NRTIs), non-NRTIs (NNRTIs), protease inhibitors (PIs), integrase strand transfer inhibitors and entry inhibitors. This was for the purpose of examining polypharmacy in PLWH that could induce a potential drug-drug interaction (DDI) between antiretrovirals and comedications, as well as to exclude people with uncertain HIV status, such as those poorly recorded or intentionally recorded for the purpose of a claim.

The data on patients who met these criteria $(n=1445)$ during the study period were extracted from the database. The patients were grouped based on their age at the time of their last hospital visit, as follows: 18-29, 30-39, $40-49,50-59,60-69$ and $\geq 70$ years.

\section{Definitions of measures}

The following patient data during the study period were extracted from the database: demographic characteristics (age, sex), diagnoses of AIDS-defining conditions, hospital admission, diagnoses of chronic comorbidities, prescriptions of antiretrovirals and prescriptions of comedications.

The AIDS-defining conditions were identified using the ICD-10 codes, which included the following: AIDS (B24), AIDS-related complex (B24), cytomegalovirus disease (B20.2/B25 except for B25.1), candidiasis (B20.4/ B37.1), encephalopathy (B22.0), wasting syndrome 
Table 1 Demographic characteristics of studied people living with HIV ( $n=1445)$

\begin{tabular}{|c|c|}
\hline & Total $(n=1445)$ \\
\hline & n (\%) \\
\hline Age (years), median (range) & $45(18-90)$ \\
\hline Male & $1306(90.4)$ \\
\hline AIDS-defining conditions* & 915 (63.3) \\
\hline \multicolumn{2}{|l|}{ Hospital admission† } \\
\hline No & $1121(77.6)$ \\
\hline Yes & $324(22.4)$ \\
\hline For an AIDS-defining condition $\ddagger$ & $120(8.3)$ \\
\hline $\begin{array}{l}\text { For non-AIDS-defining conditions } \\
\text { only§ }\end{array}$ & $196(13.6)$ \\
\hline For unknown reasonף & $8(0.6)$ \\
\hline \multicolumn{2}{|l|}{ Chronic comorbidity } \\
\hline Diabetes & $387(26.8)$ \\
\hline Hypertension & $263(18.2)$ \\
\hline Lipid disorders & $456(31.6)$ \\
\hline Vascular diseases & $55(3.8)$ \\
\hline Kidney failure & $78(5.4)$ \\
\hline Malignancies & $117(8.1)$ \\
\hline Psychiatric disorders & $219(15.2)$ \\
\hline Osteoporosis & $85(5.9)$ \\
\hline Hepatitis B/C coinfection & $263(18.2)$ \\
\hline Use of a non-antiretroviral medication ${ }^{\star \star}$ & $1086(75.2)$ \\
\hline \multicolumn{2}{|l|}{ Type of key drug among antiretrovirals } \\
\hline INSTI & 789 (54.8) \\
\hline NNRTI & 359 (24.9) \\
\hline $\mathrm{PI}$ & $617(42.8)$ \\
\hline
\end{tabular}

${ }^{*}$ Patients were considered to have an AIDS-defining condition if they had at least one disease record of AIDS-defining conditions during the study period. The only exception is for recurrent pneumonia; patients were considered to have recurrent pneumonia only when they had two or more codes of pneumonia (J12-18/ B01.2) recorded within 18 months.

†Hospital admissions for any cause during the study period. $\ddagger$ Patients with at least one record of hospital admission and whose reason for admission included an AIDS-defining condition. $\S$ Patients with at least one record of hospital admission and whose reason for admission was only non-AIDS-defining conditions. IPatients with at least one record of hospital admission and whose reason for admission was unknown (ie, no data available). ${ }^{* *}$ Non-antiretroviral medication prescribed for use for $\geq 30$ days total during the study period was taken into consideration. INSTI, integrase strand transfer inhibitor; NNRTI, non-nucleoside reverse transcriptase inhibitor; $\mathrm{PI}$, protease inhibitor.

(B22.2), coccidioidomycosis (B38.3-38.9), cryptococcosis (B45.1-45.9), cryptosporidiosis (A07.2), herpes simplex infections (B00), histoplasmosis (B39.3-39.9), isosporiasis (A07.3), pulmonary mycobacterial infection (A31.0), tuberculosis (A15-19/B20.0), mycobacterial infections (A31.8), pneumocystosis (B59, B20.6), recurrent pneumonia (J12-18/B01.2), progressive multifocal leukoencephalopathy (A81.2), salmonella septicaemia (A02.1), toxoplasma meningoencephalitis (B58.2), Burkitt lymphoma (B21.1/C83.7), Kaposi sarcoma (B21.0/C46), non-Hodgkin's lymphoma (B21.2/C82-85 except for C83.7/C91.5) and cancer of the cervix uteri (C53).

The chronic comorbidities were identified by the presence of relevant ICD-10 codes for particular diseases defined below. If the code was recorded at least once during the study period, the patients were considered to have that corresponding chronic comorbidity. These codes are recorded as disease records when patients receive diagnoses and any medical procedures, treatment or prescriptions for the disease. The chronic comorbidities in this study included type II diabetes (E11-14), hypertension (I10-15, except for I11/I13), lipid disorders (hypercholesterolaemia/hyperlipidaemia (E78.0-78.5)), vascular diseases (hypertensive heart and renal diseases (I11/I13), angina pectoris (I20), myocardial infarction (I22), stroke (I64)), kidney failure (N18-19), malignancies (B21.0-21.2/C00-97), psychiatric disorders (dementia (F01/F03), psychosis (F20-29), mania and depression (F30-32), anxiety (F40-41), insomnia (F51)), osteoporosis (M80-81) and hepatitis B/C coinfection (B18). Presence of two or more chronic comorbidities in a patient was defined as multimorbidity.

Although all malignancies were considered as one category of chronic comorbidity, we classified malignancies into two groups to further analyse them in terms of their types: (1) AIDS-defining cancers defined as Burkitt lymphoma (B21.1/C83.7), Kaposi sarcoma (B21.0/ C46), non-Hodgkin's lymphoma (B21.2/C82-85 except for C83.7/C91.5) and cancer of the cervix uteri (C53) and (2) non-AIDS-defining cancers defined as all other malignancies.

Comedication was defined as a non-antiretroviral medication prescribed for use for $\geq 30$ days total during the study period. All medications other than antiretrovirals were considered in accordance with classification based on the therapeutic subgroups (second level) of the Anatomical Therapeutic Chemical codes. ${ }^{20}$ To focus on the medications probably used for chronic treatment, or at least used for many days, instead of those used for only a short time, we set the minimum prescription days (ie, total number of days for which the particular medication was supplied irrespective of whether it was prescribed continuously or intermittently during the study period) as 30 .

All data on diseases and medications during the study period were considered as data pertaining to the PLWH and thus were counted regardless of whether they were recorded before or after the diagnosis record of HIV. This was because it was considered from a clinical perspective that the comorbidities developed before the HIV infection were persistent over the whole study period. 


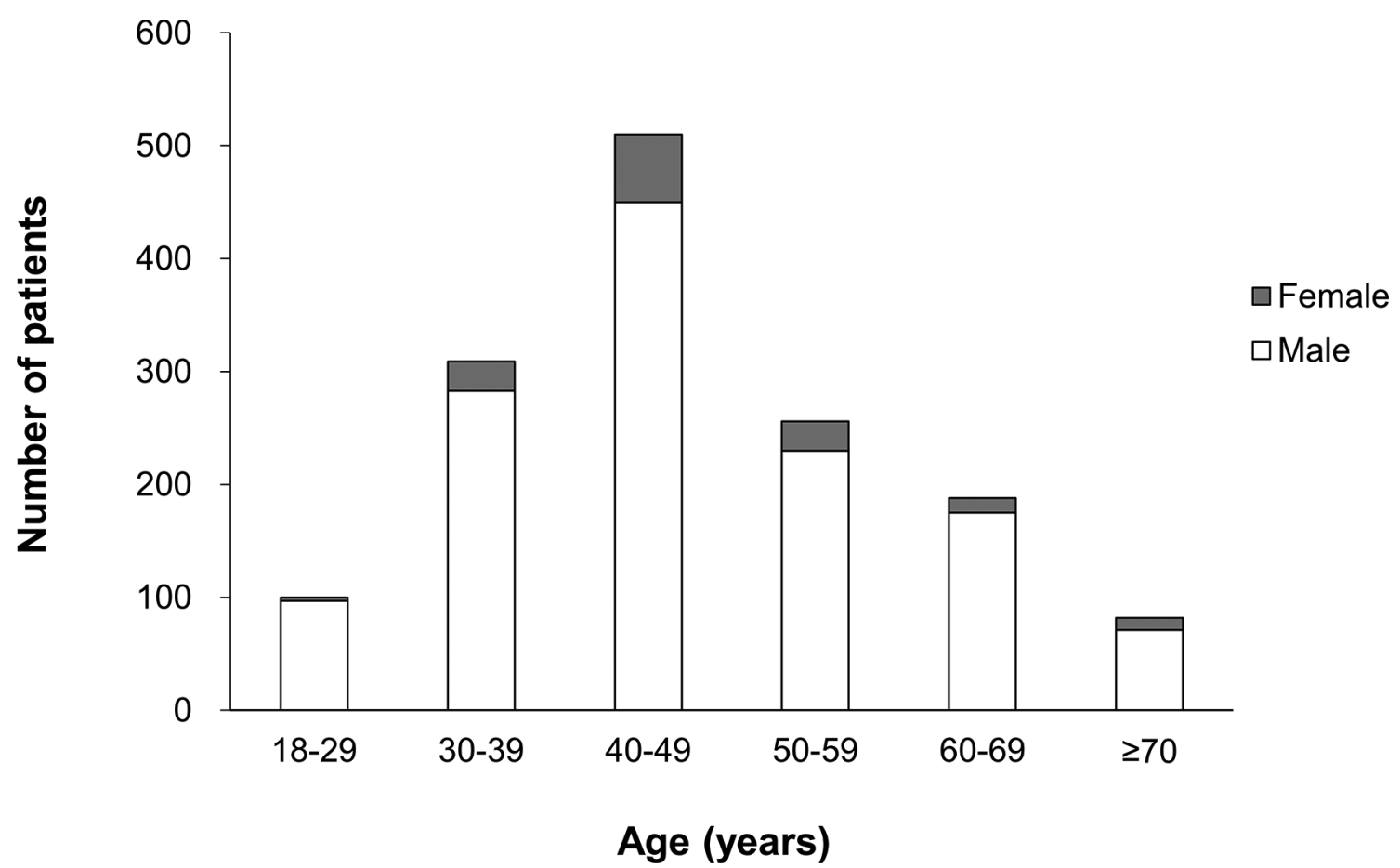

Figure 1 Age and sex distribution of studied patients living with HIV ( $n=1445)$.

\section{Patient and public involvement}

This was a hospital claims database study exclusively using pre-existing, de-identified claims data and patient recruitment was not carried out specifically for this study. Thus, no patient involvement existed in this study.

\section{Statistical analysis}

Demographic data were descriptively summarised using the median and range for continuous variables or the number and percentage (\%) of patients for categorical variables. Chronic comorbidities and comedications were descriptively summarised by age group. The $95 \%$ CIs were calculated using the exact binomial method proposed by Clopper and Pearson. ${ }^{21}$ All statistical analyses were performed using SAS V.9.4 (SAS Institute) software.

\section{RESULTS}

\section{Patient characteristics}

Of 3155 patients with a diagnosis record of HIV, 1445 $(45.8 \%)$ also had a prescription record of antiretrovirals, and were therefore included in this study. They had a median (range) age of 45 (18-90) years, and $90.4 \%$ were men (table 1). Of these patients, $915(63.3 \%)$ had an AIDS-defining condition at some point during the study period. The majority of patients $(77.6 \%)$ had only outpatient data, and $22.4 \%$ had at least one record of hospital admission for any reason during the study period (see online supplementary table 1 for a full list of non-AIDS-defining illnesses that led to hospital admission in patients who had hospital admission only for non-AIDS-defining conditions). Figure 1 shows the age and sex distribution of patients. The majority were aged $40-49$ years, followed by $30-39,50-59,60-69,18-29$ and $\geq 70$ years.

\section{Chronic comorbidities}

Overall, 1923 chronic comorbidities were reported in 972 (67.3\%) PLWH, and 523 (36.2\%) patients had multimorbidity (two or more chronic comorbidities). The most common chronic comorbidities were lipid disorders (31.6\%; $95 \%$ CI $29.2 \%$ to $34.0 \%$ ), followed by diabetes (26.8\%; $95 \%$ CI $24.5 \%$ to $29.1 \%$ ), hypertension (18.2\%; $95 \% \mathrm{CI} 16.2 \%$ to $20.3 \%$ ) and hepatitis $\mathrm{B} / \mathrm{C}$ coinfection (18.2\%; $95 \%$ CI $16.2 \%$ to $20.3 \%$ ) (table 1) (see online supplementary table 2 for the breakdown of prevalence of each disease, classified under vascular diseases and psychiatric disorders).

Figure 2A shows the number of chronic comorbidities by age. Although $58.0 \%(58 / 100)$ of patients aged 18-29 years had no chronic comorbidity, the proportions with no chronic comorbidity decreased in the older age groups. More patients in the older age groups had multimorbidity: $21.0 \%(65 / 309)$ of those aged $30-39$ years, $44.1 \%(113 / 256)$ of those aged $50-59$ years and $70.7 \%$ $(58 / 82)$ of those aged $\geq 70$ years. No patients aged $18-29$ years had four or more chronic comorbidities, while $24.4 \%(20 / 82)$ of those aged $\geq 70$ years did.

Figure 2B shows the prevalence of different types of chronic comorbidities by age group. The prevalence of most chronic comorbidities tended to be higher in the older age groups, but such trends were not observed for psychiatric disorders (prevalence range: 12.0\%-17.8\%; $\mathrm{p}=0.3271$, Cochran-Armitage trend test) and hepatitis $\mathrm{B} / \mathrm{C}$ coinfection $(17.1 \%-20.4 \%, \mathrm{p}=0.4501$, Cochran-Armitage trend test). The most common chronic comorbidities in the older age groups were hypertension, diabetes and lipid disorders. Hypertension was the most common chronic comorbidity in the $\geq 70$ years group $(52.4 \%$; 


\section{A) Number of chronic comorbidities by age group}

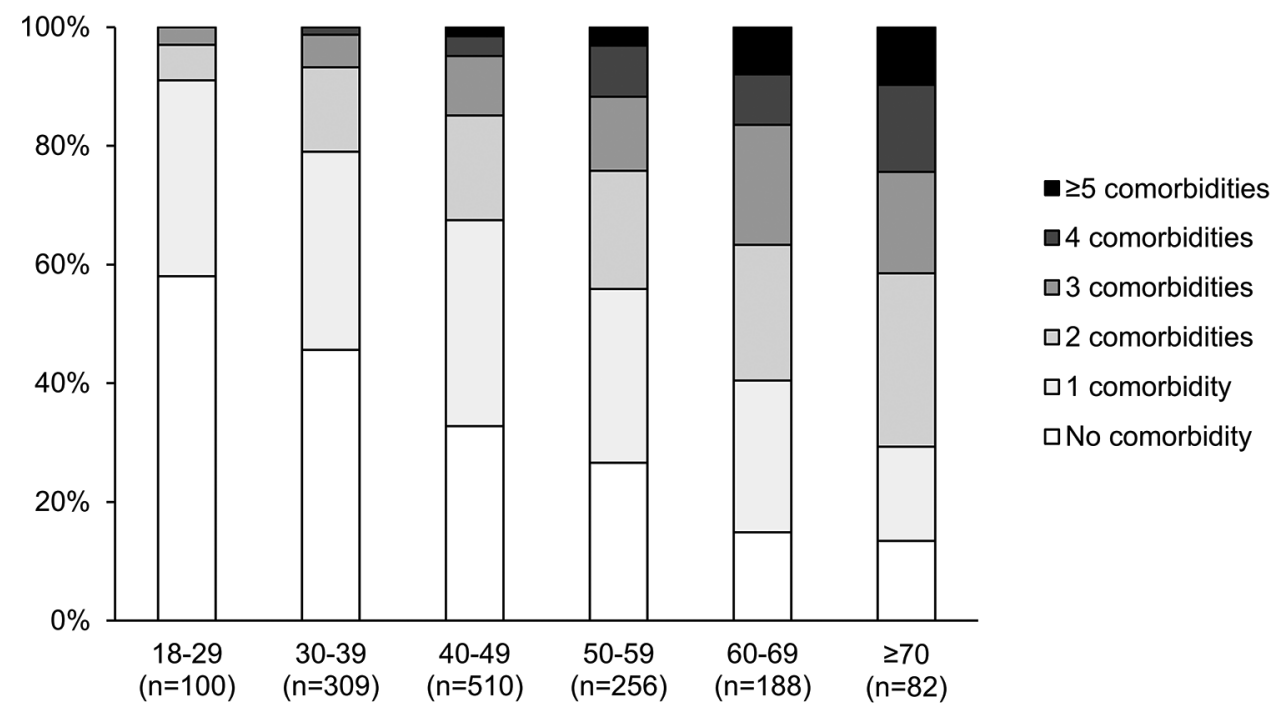

Age (years)

\section{B) Type of chronic comorbidities by age group}

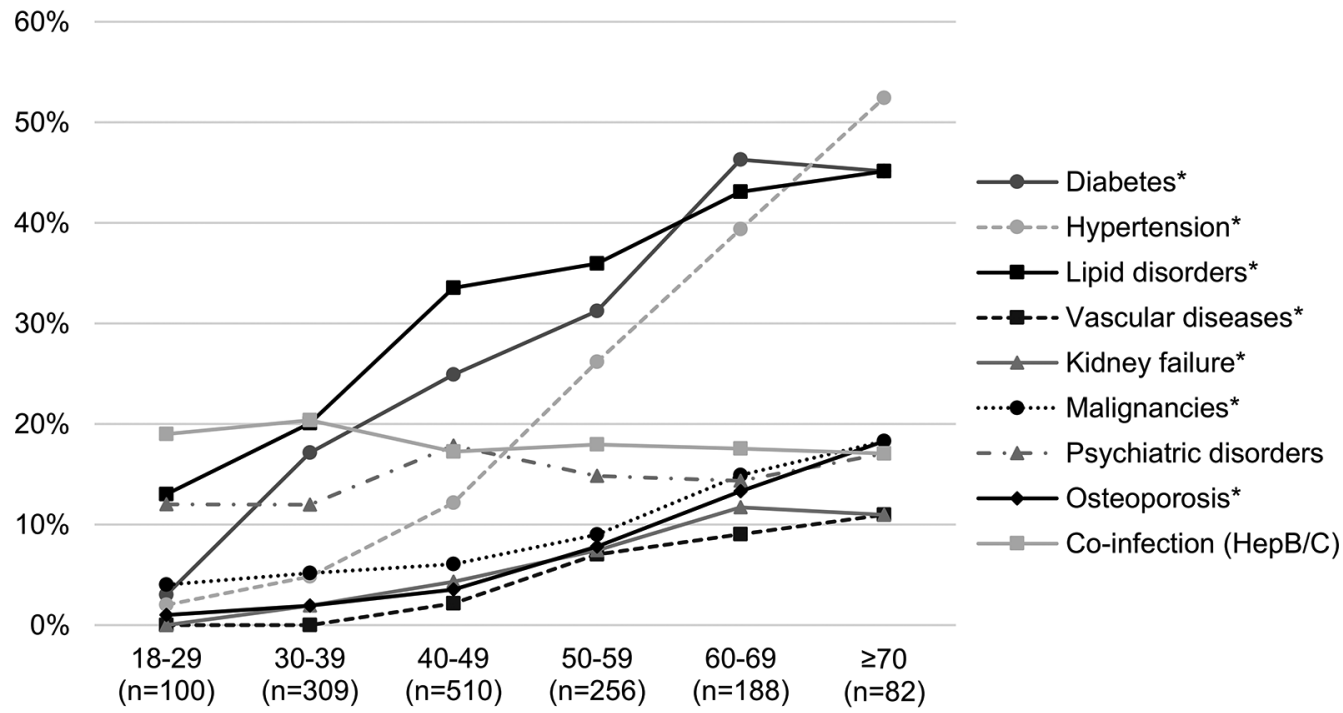

Age (years)

Figure 2 Number and type of chronic comorbidities by age group. ${ }^{*} \mathrm{P}<0.0001$ under the Cochran-Armitage trend test.

$95 \%$ CI $41.1 \%$ to $63.6 \%$ ), diabetes in the $60-69$ years group (46.3\%; 95\% CI $39.0 \%$ to $53.7 \%$ ) and lipid disorders in the 50-59years group (35.9\%; 95\% CI $30.1 \%$ to $42.1 \%$ ). The prevalence of vascular diseases, kidney failure, malignancies or osteoporosis in the $\geq 70$ years group was $11.0 \%-18.3 \%$, which was approximately $10 \%-15 \%$ higher than its counterpart in the 18-29years group $(0 \%-4.0 \%) \quad(\mathrm{p}<0.0001$ for all four comorbidity types, Cochran-Armitage trend test).

\section{Comedications}

Of the 1445 PLWH, 1086 (75.2\%) used at least one comedication and $342(23.7 \%)$ used five or more, apart from antiretrovirals. Figure 3A shows the number of comedications (excluding antiretrovirals) by age group. The proportions of patients who used at least one comedication were $53.0 \%(53 / 100)$ in the $18-29$ years group, $76.1 \%$ $(388 / 510)$ in the $40-49$ years group and $86.6 \%(71 / 82)$ in the $\geq 70$ years group. More patients in the older age 
A) Number of co-medications by age group

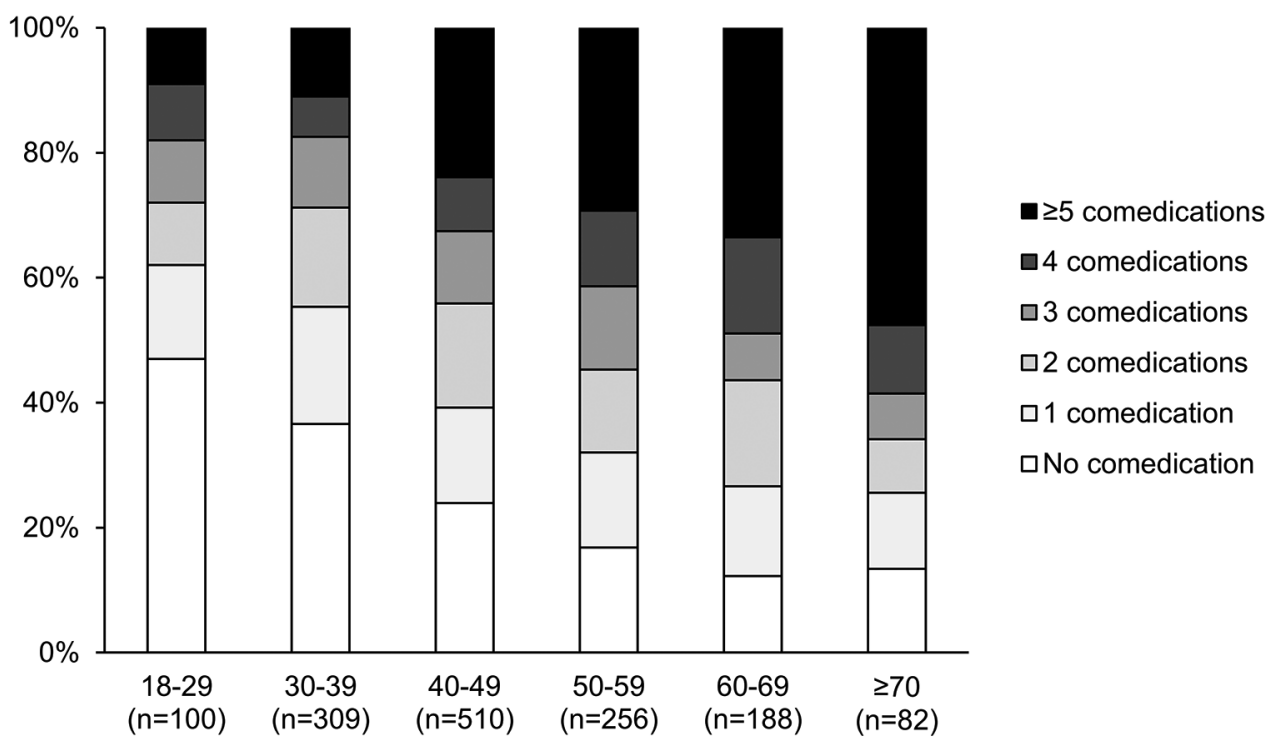

Age (years)

\section{B) Type of co-medications by age group}

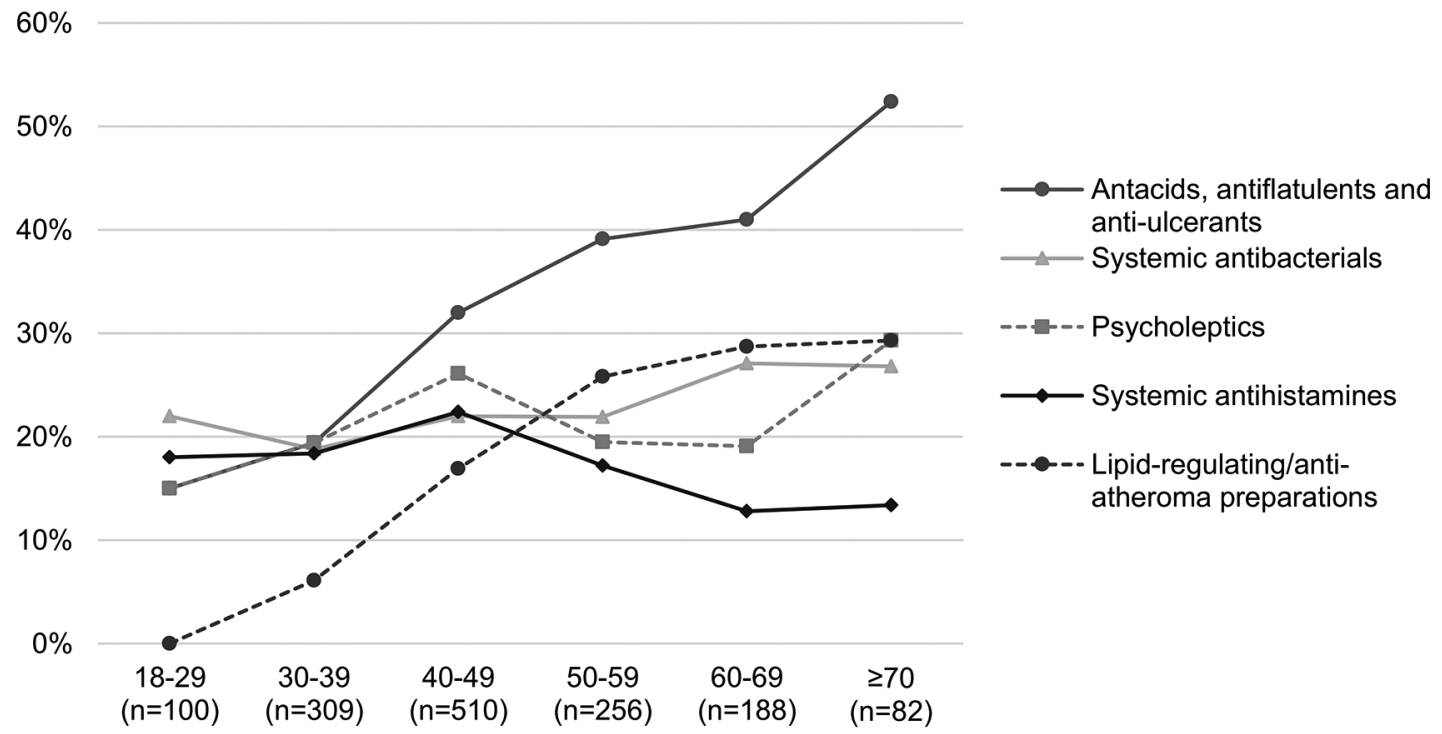

Age (years)

Figure 3 (A) Number of comedications prescribed by age group. (B) The top five common comedications by age group.

groups used greater numbers of comedications: $11.0 \%$ $(34 / 309)$ of patients in the 30-39years group, $29.3 \%$ $(75 / 256)$ in the $50-59$ years group and $47.6 \%(39 / 82)$ in the $\geq 70$ years group used five or more comedications.

The 10 most common therapeutic categories were antacids, antiflatulents and antiulcerants (A02) (31.7\%), systemic antibacterials (J01) (22.2\%), psycholeptics (N05) (22.0\%), systemic antihistamines (R06) (18.5\%), lipid-regulating/antiatheroma preparations (C10)
(17.2\%), anti-inflammatory and antirheumatic products (M01) (15.5\%), antidiarrhoeals, oral electrolyte replacers and intestinal anti-inflammatories (A07) $(12.1 \%)$, agents acting on the renin-angiotensin system (C09) (11.1\%), vitamins (A11) (10.2\%); and cough and cold preparations (R05) (9.6\%). Regarding the top five most common comedications, the proportions of patients who took respective comedications by age group are shown in figure 3B. Antacids, antiflatulents and antiulcerants were 


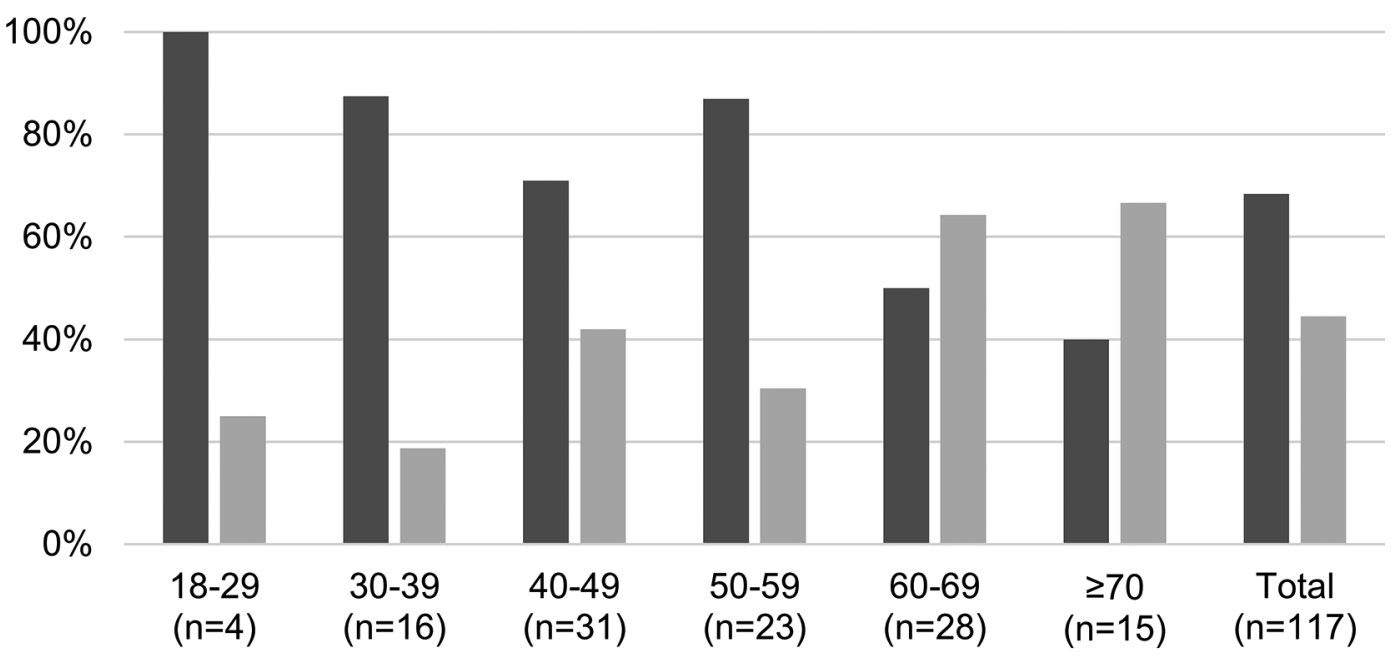

Age group

- With AIDS-defining cancers $\quad$ With non-AIDS-defining cancers

Figure 4 Proportions of studied patients with AIDS-defining cancers and non-AIDS-defining cancers among patients with any malignancies within each age group.

the most common in most of the age groups, notably in the $\geq 70$ years group $(52.4 \%)$. The proportions of patients taking systemic antibacterials, psycholeptics or systemic antihistamines were similar across all age groups. Lipid-regulating agents were used more commonly in the older age groups: notably in $25 \%-30 \%$ of patients aged $\geq 50$ years.

\section{Malignancies}

In this study, 151 malignancies were reported in 117 PLWH, including 84 AIDS-defining cancers and 67 non-AIDS-defining cancers. Figure 4 shows the proportions of patients with AIDS-defining cancers and those of patients with non-AIDS-defining cancers among patients with any malignancies within each age group. The figure illustrates that AIDS-defining cancers were more common in people $<60$ years, whereas non-AIDS-defining cancers were more frequent in people aged $\geq 60$ years. Table 2 shows the proportions of patients who had respective cancer types in these 117 PLWH with any malignancies. Non-Hodgkin's lymphoma was the most common type of AIDS-defining cancer (52.1\%), and bronchus or lung cancers were the most common types of non-AIDS-defining cancers $(6.8 \%)$.

\section{DISCUSSION}

This is the first study to investigate the prevalence of chronic comorbidities and the use of comedications in PLWH in Japan taking antiretrovirals, using a large hospital claims database. The study found that chronic comorbidities were common in PLWH in Japan, particularly hypertension, diabetes and lipid disorders among older patients. In terms of burden by age group, this study revealed that older patients had greater numbers of chronic comorbidities and also used greater numbers of comedications than younger patients did.

Greater numbers of comorbidities in older people with HIV were also reported in previous international studies. ${ }^{2}{ }^{22}$ Additionally, older people with HIV in Switzerland and the USA were more likely to have diabetes mellitus, cardiovascular diseases, non-AIDS-defining malignancies, osteoporosis or liver diseases than younger patients. ${ }^{32}$ Similar to those studies, in the present study, diabetes mellitus, vascular diseases and osteoporosis were more common in older PLWH. Kidney failure was also more common in older patients, as suggested by a previous study reporting that both HIV infection and increased age were associated with an increased risk of kidney diseases in PLWH. ${ }^{23}$ Previous studies also showed PLWH had a greater number of age-associated comorbidities at earlier ages than those living without HIV, suggesting premature ageing in PLWH. ${ }^{26}$ This is a topic we plan to examine in our future research using a control group of people without HIV.

Despite the decreased incidence of non-Hodgkin's lymphoma and Kaposi sarcoma, owing to antiretrovirals, ${ }^{24} 25$ AIDS-defining cancers remain a great concern in PLWH in North America. ${ }^{26}$ However, in the present study, these cancers were not commonly observed (non-Hodgkin's lymphoma, 4.2\% (61/1445); Kaposi sarcoma, 1.1\% $(16 / 1445))$. Although direct comparison is not appropriate because of different study methods (ie, different study populations, different antiretroviral eras), the prevalence was much lower than that reported in a previous Japanese study in which non-Hodgkin's lymphoma and Kaposi sarcoma were present in $37.9 \%$ and $15.2 \%$, respectively, of autopsied people with HIV and having received antiretrovirals from 1985 to $2012 .{ }^{27}$ Another interesting 
Table 2 Proportions of studied patients with respective cancer types among people living with HIV who had any malignancies $(n=117)$

\begin{tabular}{|c|c|}
\hline & $\begin{array}{l}\text { Patients with } \\
\text { malignancy } \\
(n=117)\end{array}$ \\
\hline & n (\%) \\
\hline \multicolumn{2}{|l|}{ AIDS-defining cancer } \\
\hline Non-Hodgkin's lymphoma & $61(52.1)$ \\
\hline Kaposi sarcoma & $16(13.7)$ \\
\hline Burkitt lymphoma & $5(4.3)$ \\
\hline Cervix uteri & $2(1.7)$ \\
\hline \multicolumn{2}{|l|}{ Non-AIDS-defining cancer } \\
\hline Bronchus or lung & $8(6.8)$ \\
\hline $\begin{array}{l}\text { Secondary malignant neoplasm of bone } \\
\text { and bone marrow }\end{array}$ & $6(5.1)$ \\
\hline Colon & $3(2.6)$ \\
\hline Breast & $3(2.6)$ \\
\hline $\begin{array}{l}\text { Malignant neoplasm, without } \\
\text { specification of site }\end{array}$ & $3(2.6)$ \\
\hline $\begin{array}{l}\text { Secondary malignant neoplasm of brain } \\
\text { and cerebral meninges }\end{array}$ & $3(2.6)$ \\
\hline Acute myeloblastic leukaemia & $3(2.6)$ \\
\hline Multiple myeloma & $3(2.6)$ \\
\hline Stomach & $2(1.7)$ \\
\hline Secondary malignant neoplasm of lung & $2(1.7)$ \\
\hline Liver cell carcinoma & $2(1.7)$ \\
\hline Sigmoid colon & $2(1.7)$ \\
\hline Thoracic part of oesophagus & $2(1.7)$ \\
\hline Skin of trunk & $2(1.7)$ \\
\hline Others $^{\star}$ & $23(19.7)$ \\
\hline
\end{tabular}

*Non-AIDS-defining cancer types present only in one patient were grouped and presented as 'others'.

finding of the present study was that non-AIDS-defining cancers, which accounted for $44.4 \%$ of all malignancies, seemed more frequent in patients in older age groups, being observed in $>60 \%$ of PLWH aged $\geq 60$ years who had any malignancies. A previous study showed that age-related cancers, such as lung, prostate, colorectal and breast cancers, were common among older PLWH. ${ }^{28}$ Another study also reported an increase in non-AIDS-defining cancers among the HIV population as a consequence of ageing of the AIDS population. ${ }^{24}$ In light of these previous findings, our results highlight the importance of non-AIDS-defining cancers among PLWH because of their extended lifespans. However, in the present study, it was difficult to illuminate any trends in the prevalence of malignancies by age group because of the small number of patients with malignancies. Thus, the prevalence of malignancies by age and cancer type should be interpreted carefully, and examined further in future studies.
Our results indicated that use of comedications was common among older PLWH, although the proportions of patients with five or more comedications in those aged $\geq 50$ years were smaller $(33.7 \%)$ compared with a previous study $(54 \%),{ }^{29}$ suggesting the management of older PLWH may be complicated because of DDIs attributed to increased use of comedications. ${ }^{30}{ }^{31}$ Moreover, in our study the use of comedications was common even in younger PLWH, and over half of patients aged 18-29 years used at least one comedication. These results suggest the importance of potential DDIs among the older PLWH and the younger ones. For instance, DDIs between PIs or NNRTIs and comedications (eg, cardiovascular agents, antiplatelet agents, psychotropics, antidepressants, analgesics and methadone) can be common in PLWH. ${ }^{30} 31$ The common use of lipid-regulating agents and agents acting on the renin-angiotensin system in the present study probably reflected the high prevalence of lipid disorders and hypertension, especially in older patients, for some of whom antiretrovirals may be partly responsible for changes in lipid metabolism, ${ }^{32} 33$ although its underlying mechanisms in PLWH are complex. Psycholeptics were also commonly used irrespective of age, which was consistent with previous studies, ${ }^{30} 31$ and probably due to HIV disease progression and treatment with antiretrovirals associated with psychiatric disorders. ${ }^{34} 35$ The most common use, of antacids, antiflatulents and antiulcerants $(31.7 \%)$, may partly reflect the common presence of gastric ulcers in hospitalised older Japanese patients, ${ }^{36}$ although not all of our study population had hospitalisation. Because antacids can reduce serum concentrations of several antiretrovirals by altering the acid environment, ${ }^{37} 38$ Japanese physicians need to pay careful attention when prescribing antiretrovirals to patients using them.

The present study has some limitations. Because it was a cross-sectional study, chronic comorbidities/comedications were not examined against the timing of a diagnosis of HIV infection. It should be noted that chronic comorbidities/comedications in this study included those coded before a diagnosis record of HIV infection. This study is limited in its ability to draw valid conclusions about possible causality between HIV infection and chronic comorbidities/comedications; therefore, causality should be confirmed through more rigorous studies. Other limitations include those inherent to observational studies using a hospital claims database, such as poorly recorded or missing information, which may result in misclassification. For example, because the database is hospital based, medical/treatment history recorded at different hospitals could not be obtained, meaning that patients may have received comedications for other diseases at different hospitals. Acute conditions may be misclassified into chronic comorbidities, as these were defined only by the presence of one relevant ICD-10 code. Comedications only for treating acute condition may contaminate comedications prescribed only for chronic medical conditions, 
although medications prescribed for $<30$ days were excluded from comedications in this study. Additionally, the study results may not be generalisable to the overall population living with HIV and taking antiretrovirals in Japan. This is because PLWH in this database who require acute care at hospitals covered in the database may be more ill than other PLWH who do not require acute care, or those who receive HIV treatment at non-acute care hospitals not covered in the database. In addition, the study results, particularly results on comedications, may not accurately represent comorbidity and comedication profiles in the general population living with HIV. This is because this study did not investigate these profiles for inpatients separately from those for outpatients. Online supplementary table 3 shows comedication lists by patients with or without a hospital admission record during the study period. Comedication results may also over-represent drugs used by PLWH who sought hospital care, which may differ from those of PLWH who do not seek hospital care. HIV treatment choices in these included hospitals may not be representative of those made in other hospitals (ie, hospitals not providing advanced medical treatment). This study excluded patients without a record of antiretrovirals, who represent PLWH not taking antiretrovirals. However, we might have excluded some PLWH with ART because PLWH in this database may have received antiretrovirals at hospitals not covered in the database. Finally, because detailed data regarding HIV infection status were not available, we could not analyse the potential relationships between chronic comorbidities and CD4 count, HIV-RNA or ART duration.

In conclusion, chronic comorbidities and comedications were common among PLWH in Japan taking antiretrovirals, especially among older patients, who more frequently used comedications. This suggests the need for special treatment and attention by physicians. Although our results, particularly the types of comedications, may not necessarily be extrapolated to a different patient population, they will help remind Japanese physicians of the importance of being aware of such complicated medical profiles of PLWH, especially patients who seek treatment at a hospital, so as to treat these people while mindful of potential DDIs. Application of our results can support the development of optimal healthcare strategies for this growing population.

Acknowledgements Medical writing and statistical supports were provided by Tetsumi Toyoda and Yuri Haga of Clinical Study Support. We are particularly grateful for the assistance given by Noriyo Ihara of MSD K.K.

Contributors DJR contributed to the study design. DJR, KI and KT contributed to the data analysis and interpretation. DJR, KI, KT and TN revised the manuscript draft critically and approved the final version of the manuscript to be published.

Funding This study was supported by MSD K.K., Tokyo, Japan.

Competing interests DJR, Kl and KT are employees of MSD K.K., Tokyo, Japan. TN has received no funding for this study but received lecture fees from MSD K.K., Tokyo, Japan.

Patient consent Not required.
Ethics approval The requirement for informed consent was waived because this was an observational study using de-identified claims data and the Japanese Ethical Guidelines for Medical and Health Research Involving Human Subjects do not apply to a study exclusively using de-identified data.

Provenance and peer review Not commissioned; externally peer reviewed. Data sharing statement № additional data are available.

Open access This is an open access article distributed in accordance with the Creative Commons Attribution Non Commercial (CC BY-NC 4.0) license, which permits others to distribute, remix, adapt, build upon this work non-commercially, and license their derivative works on different terms, provided the original work is properly cited and the use is non-commercial. See: http://creativecommons.org/ licenses/by-nc/4.0/

(c) Article author(s) (or their employer(s) unless otherwise stated in the text of the article) 2018. All rights reserved. No commercial use is permitted unless otherwise expressly granted.

\section{REFERENCES}

1. Nakagawa F, May M, Phillips A. Life expectancy living with HIV: recent estimates and future implications. Curr Opin Infect Dis 2013;26:17-25.

2. Guaraldi G, Orlando G, Zona S, et al. Premature age-related comorbidities among HIV-infected persons compared with the general population. Clin Infect Dis 2011;53:1120-6.

3. Hasse $\mathrm{B}$, Ledergerber $\mathrm{B}$, Furrer $\mathrm{H}$, et al. Morbidity and aging in HIV-infected persons: the Swiss HIV cohort study. Clin Infect Dis 2011;53:1130-9.

4. Wu PY, Chen MY, Hsieh SM, et al. Comorbidities among the HIV-infected patients aged 40 years or older in Taiwan. PLoS One 2014;9:e104945.

5. Vance DE, Mugavero M, Willig J, et al. Aging with HIV: a crosssectional study of comorbidity prevalence and clinical characteristics across decades of life. J Assoc Nurses AIDS Care 2011;22:17-25.

6. Schouten J, Wit FW, Stolte IG, et al. Cross-sectional comparison of the prevalence of age-associated comorbidities and their risk factors between HIV-infected and uninfected individuals: the AGEhIV cohort study. Clin Infect Dis 2014;59:1787-97.

7. Mangoni AA, Jackson SH. Age-related changes in pharmacokinetics and pharmacodynamics: basic principles and practical applications. Br J Clin Pharmacol 2004:57:6-14.

8. Edelman EJ, Gordon KS, Glover J, et al. The next therapeutic challenge in HIV: polypharmacy. Drugs Aging 2013;30:613-28.

9. Seaberg EC, Muñoz A, Lu M, et al. Association between highly active antiretroviral therapy and hypertension in a large cohort of men followed from 1984 to 2003. AIDS 2005;19:953-60.

10. Deeks SG, Phillips AN. HIV infection, antiretroviral treatment, ageing, and non-AIDS related morbidity. BMJ 2009;338:a3172.

11. Deeks SG. HIV infection, inflammation, immunosenescence, and aging. Annu Rev Med 2011;62:141-55.

12. Wing EJ. HIV and aging. Int $J$ Infect Dis 2016;53:61-8.

13. Weber R, Sabin CA, Friis-Møller N, et al. Liver-related deaths in persons infected with the human immunodeficiency virus: the D:A:D study. Arch Intern Med 2006;166:1632-41.

14. Sackoff JE, Hanna DB, Pfeiffer MR, et al. Causes of death among persons with AIDS in the era of highly active antiretroviral therapy: New York City. Ann Intern Med 2006;145:397.

15. World Health Organization. Global Health Observatory (GHO) Data. 2016. http://www.who.int/gho/publications/world_health_statistics/ 2016/en/ (accessed 19 Mar 2017).

16. Anon. Trends in AIDS occurrence report. 2015. In Japanese http:// api-net.jfap.or.jp/status/2015/15nenpo/15nenpo_menu.html (accessed 19 Mar 2017)

17. Costagliola D. Demographics of HIV and aging. Curr Opin HIV AIDS 2014;9:294.301.

18. Yanagimoto S, Yotsuyanagi H, Kikuchi Y, et al. Chronic hepatitis $B$ in patients coinfected with human immunodeficiency virus in Japan: a retrospective multicenter analysis. J Infect Chemother 2012;18:883.90.

19. Yanagisawa $\mathrm{N}$, Ando M, Ajisawa A, et al. Clinical characteristics of kidney disease in Japanese HIV-infected patients. Nephron Clin Pract 2011;118:c285-91.

20. EphMRA. EphMRA anatomical classification guidelines. http://www. ephmra.org/user_uploads/atcguidelines2016final.pdf (accessed 24 May 2017).

21. Clopper CJ, Pearson ES. The use of confidence or fiducial limits illustrated in the case of the binomial. Biometrika 1934;26:404-13. 
22. Goulet JL, Fultz SL, Rimland D, et al. Aging and infectious diseases: do patterns of comorbidity vary by HIV status, age, and HIV severity? Clin Infect Dis 2007;45:1593-601.

23. Hirschhorn LR, Kaaya SF, Garrity PS, et al. Cancer and the 'other' noncommunicable chronic diseases in older people living with HIV/ AIDS in resource-limited settings: a challenge to success. AIDS 2012;26:S65-75.

24. Shiels MS, Pfeiffer RM, Gail MH, et al. Cancer burden in the HIV-infected population in the United States. J Nat/ Cancer Inst 2011;103:753-62.

25. Patel P, Hanson DL, Sullivan PS, et al. Incidence of types of cancer among HIV-infected persons compared with the general population in the United States, 1992-2003. Ann Intern Med 2008;148:728-36.

26. Silverberg MJ, Lau B, Achenbach CJ, et al. Cumulative incidence of cancer among persons with HIV in North America: a cohort study. Ann Intern Med 2015;163:507.18.

27. Katano H, Hishima T, Mochizuki M, et al. The prevalence of opportunistic infections and malignancies in autopsied patients with human immunodeficiency virus infection in Japan. BMC Infect Dis 2014;14:229.

28. Yanik EL, Katki HA, Engels EA. Cancer risk among the HIV-infected elderly in the United States. AIDS 2016;30:1663-8.

29. Holtzman C, Armon C, Tedaldi E, et al. Polypharmacy and risk of antiretroviral drug interactions among the aging HIV-infected population. J Gen Intern Med 2013;28:1302-10.
30. Tseng A, Szadkowski L, Walmsley S, et al. Association of age with polypharmacy and risk of drug interactions with antiretroviral medications in HIV-positive patients. Ann Pharmacother 2013:47:1429-39.

31. Marzolini C, Back D, Weber R, et al. Ageing with HIV: medication use and risk for potential drug-drug interactions. J Antimicrob Chemother 2011;66:2107-11.

32. da Cunha J, Maselli LM, Stern AC, et al. Impact of antiretroviral therapy on lipid metabolism of human immunodeficiency virusinfected patients: Old and new drugs. World J Virol 2015;4:56-77.

33. Lake JE, Currier JS. Metabolic disease in HIV infection. Lancet Infect Dis 2013;13:964-75.

34. Brogan K, Lux J. Management of common psychiatric conditions in the HIV-positive population. Curr HIVIAIDS Rep 2009;6:108-15.

35. Watkins CC, Treisman GJ. Neuropsychiatric complications of aging with HIV. J Neurovirol 2012;18:277-90.

36. Mizokami F, Koide Y, Noro T, et al. Polypharmacy with common diseases in hospitalized elderly patients. Am J Geriatr Pharmacother 2012;10:123-8.

37. de Maat MM, Ekhart GC, Huitema AD, et al. Drug interactions between antiretroviral drugs and comedicated agents. Clin Pharmacokinet 2003;42:223-82.

38. Nachega JB, Hsu AJ, Uthman OA, et al. Antiretroviral therapy adherence and drug-drug interactions in the aging HIV population. AIDS 2012;26:S39-53. 